Article

\title{
Personal Power and Agency When Dealing with Interactive Voice Response Systems and Alternative Modalities
}

\author{
Jill Walsh, Brittany Leigh Andersen *, James E. Katz and Jacob Groshek \\ Division of Emerging Media Studies, Boston University, Boston, MA 02215 USA; E-Mails: jillw@bu.edu (J.W.), bla@bu.edu \\ (B.A.), katz2020@bu.edu (J.K.), jgroshek@bu.edu (J.G.)
}

* Corresponding author

Submitted: 1 October 2017 | Accepted: 16 March 2018 | Published: 25 September 2018

\begin{abstract}
In summer 2015, we conducted an exploratory study of how people in the U.S. use and respond to robot-like systems in order to achieve their needs through mediated customer service interfaces. To understand this process, we carried out three focus groups sessions along with 50 in-depth interviews. Strikingly we found that people perceive (correctly or not) that interactive voice response customer service technology is set up to deter them from pursuing further contact. And yet, for the most part, people were unwilling to simply give up on the goals that motivated their initial contact. Consequently, they had to innovate ways to communicate with the automated systems that essentially serve as gatekeepers to their desired ends. These results have implications for communication theory and system design, especially since these systems will be increasingly presented to consumers as social media affordances evolve.
\end{abstract}

\section{Keywords}

computer mediated communication (CMC); interactive voice response systems (IVRs); media equation theory; power in communication; social robots; theory of mind

\section{Issue}

This article is part of a multidisciplinary issue of Media and Communication, edited by Epp Lauk (University of Jyväskylä, Finland) and Raul Reis (Emerson College, USA).

(C) 2018 by the authors; licensee Cogitatio (Lisbon, Portugal). This article is licensed under a Creative Commons Attribution 4.0 International License (CC BY).

\section{Introduction}

Interactive voice response systems (IVRs) and other robot-like technology are used widely by corporations and other institutions as a means to address their clients' service and information needs. IVRs are automated systems that allow a user to interact with a robot remotely, via the phone or computer. IVR robots are often used in customer service to moderate standardized and repetitive inquiries, such as with appointment scheduling, refilling prescriptions, insurance questions, or collecting bank account information. IVR systems prompt users with specific questions and direct them to different menus options based on the voice or keypad input. As such, IVRs are set up as gatekeepers to information; users must answer questions and/or provide information in order to get to the help they seek. In an effort to further un- derstand user interactions with IVR technology, we conducted an exploratory study of U.S. uses of IVR and related systems in the fall of 2015 to get deeper insight into how people communicate with power in this institutional context. Though not often a subject of academic study, this process is one that affects millions of people daily in the U.S., and as modern communication technologies spread, it will increasingly do so around the world.

Providing clients with such inbound communication opportunities, and dealing with the demands that often follow in the wake of them, are resource consumptive for organizations, and thus they have an incentive to automate and cheaply channel such services. Specifically, from an institutional perspective, using IVRs as gatekeepers makes financial sense as it reduces the need for paid employees to manage phone lines, and in theory, the wait-time for the client. Institutions can also argue that 
IVRs deliver in many cases superior customer service, leading to more satisfied clients. As a result, IVRs are almost a universal component of the client service experience and can be found in a gamut of industries, organizations, and institutions. Yet unsurprisingly, only $3 \%$ of respondents in our 2015 study reported that they liked using IVRs as part of their client service experience (Center for Research on the Information Society, 2015). Clients are thus faced with a dilemma; they need help from the institutions but dislike IVR interactions, yet despite their dislike, they are routinely forced to engage with the system to achieve their desired ends.

This conundrum in essence allows us to confront a major theme of scholarship concerning politics and communication technology, namely communicating with power. When clients contact agencies with a problem, either to request information or to address a need, they are cast in the position of a supplicant as the institution has the power to take action or refrain from doing so. Knowledge is also unequal as the institution has thorough information about what can be done to help the client and in many cases a trove of personal information about the client. Though the communicational power asymmetries are clear under the circumstances, they need not be exaggerated either. For it is also the case, based on research presented here and that of others, that people are resourceful in negotiating a set of institutional hurdles to pursue their own interests. Specifically, to manage dealing with restricted access to institutional resources that is forced upon them through automatic systems, people invoke strategic decision-making in response to the IVR gatekeeping regimes they encounter. The extant research literature covers some structured lab experiments (e.g., Groom et al., 2011) and older public opinion data (Katz, Aspden, \& Reich, 1997) about how people manage client service. Our search of the literature reveals no instances of a nuanced interpretation of these processes despite the fact that millions of people engage in them every day.

Our purpose therefore is to complement the research literature by exploring the innovative ways people use to manage their relatively and sometimes nearly absolutely powerless position in communicating via IVR client service interfaces. This work is important because in addition to explaining the client service experience it also serves as an interesting example of how people approach technological structures of power and express their human agency when dealing with automatic entities that can often render them essentially powerless. Thus it connects with another set of literature, in this case massive, concerning individual lives in relationship to institutional power structures (e.g., Castells, 2009; Foucault, 1984) but due to space limitations can only be touched upon.

\section{Powerful Communications in Review}

Ultimately, customer service contacts are negotiations between unequal players with asymmetrical knowledge.
Foucault (1984) argued that "power is everywhere; not because it embraces everything, but because it comes from everywhere" (p. 93). He goes on to say that power is not "acquired, seized, or shared" but rather something that is "exercised" in unequal relationships (p. 94). Castells (2009) is especially helpful in understanding the ways in which clients negotiate this process. Like Foucault, Castells believes that power is everywhere, but he defines it as the "relational capacity that enables a social actor to influence asymmetrically the decisions of other social actor(s) in ways that favor the empowered actor's will, interest and values" (2009, p. 10). Through these mechanisms, power is embedded in relationships rather than innate characteristics of the actors or groups (van Dijk, 2010).

This is a critical component of the customer service experience as it is the relationship between the IVR and client, in particular the IVRs' location as gatekeeper in this process, that gives it such power. We simply cannot receive a response to our question without the IVR. Castells (2009) uses the term network power to refer to a power relationship in networked communication in which protocols dictate the process. Network power is critical customer service interactions as clients are beholden to the protocols devised by the programmers and are not able to vary their communication style. This is especially important as the protocols are specifically designed to limit client interaction and in essence make the client go away.

\subsection{Cooperating with Customer Service Systems}

The notion that people respond socially to computers has been well documented in laboratory settings (Halpern \& Katz, 2013; Nass \& Brave, 2005; Nass, Fogg, \& Moon, 1996; Reeves \& Nass, 1996) and through survey research (Katz \& Halpern, 2013). As the brain cannot make distinctions between speaking with a robot or human, people end up "applying the same rules and shortcuts that they use when interacting with people" (Nass \& Brave, 2005, p. 4). Indeed, the social response appears to be automatic in people, triggered simply by a voice speaking to us, even if it is a very synthetic voice (Nass \& Brave, 2005). This would suggest that people interacting with IVRs for client service help will be inclined to follow social norms and engage in polite interactions. Of further interest for this work, it appears that when subjects are told they are working with the computer, they begin to "affiliate with the computer as a team" (Nass et al., 1996). One could imagine a situation where client service may feel like teamwork; the client and IVR do have to work together to some extent to ensure there is resolution to the problem. Interestingly, when users are asked about this, they deny these social behaviors (Nass \& Moon, 2000).

Power is embedded in the client service experience in an interesting way that may add to the need to engage in impression management work (Goffman, 1959) with the IVRs. According to the survey conducted by the 
Center for Research on the Information Society (2015), $90 \%$ of respondents want to speak to a live client service agent when they begin their experience. As IVRs are the gatekeepers to the live agent, people need to engage the technology to get to the agent they seek. Work by Fairhurst and Chandler (1989) and Tjosvold (1984) indicate the value of cooperation and smooth social interactions for affecting positive outcomes in negotiations. This data suggest that it may be in the client's best interest to employ impression management work to engage in social norms of politeness with the IVR in order to curry favor as they would with a person. Given that they are in a position of limited power, individuals can use social norms as a means to create power in these interactions. This led to the following research question:

RQ1: Will users' behavior with IVRs change based on their perceived amount of power?

There are significant communicational interaction ramifications for interacting with IVRs in this manner. First, we would assume that if people unconsciously view their experiences with IVRs as a social interaction, they will then concern themselves with the presentation of self and impression management work that is a critical component of social interactions (Goffman, 1959). When research suggests that people are polite to computers or robots (Reeves \& Nass, 1996), it is of course because of social norms about how we treat people as the authors indicate, but it is also due to our concerns about selfpresentation. We perform on the public front stage (Goffman, 1959) as we believe is expected of us to ensure that we are regarded positively by others. If the work highlighted above by Nass and his colleagues is true, then the interactions with IVRs, automated systems like robots, should provoke the same internal desires for impression management that we see in these offline interactions highlighted by Goffman. Thus, the following hypothesis is drawn:

H1: Users interacting with IVRs for client service help will be inclined to follow social norms and engage in polite interactions.

\subsection{The Theory of Mind in Computer Interactions}

One of the ways that people manage social interactions is to employ theory of mind (Premack \& Woodruff, 1978) techniques, which enable us to assign certain qualities and attributes to others. Using theory of mind helps us to feel that we can understand the feelings, knowledge, and perspective of others. We do this intuitively, as we "naturally explain people's behavior on the basis of their minds; their knowledge, their beliefs, and their desires, and we know that when there is a conflict between belief and reality it is the person's belief, not the reality that will determine their behavior" (Frith \& Frith, 2005, p. R644). This is helpful as it enables us to understand and predict the other's behavior and manage the uncertainties associated with social interactions.

Although we have the innate ability to engage theory of mind, we are not all universally adept at it (Lyons, Caldwell, \& Shultz, 2010). Importantly though, people believe that they are quite good at it and for the most part we enter into our interactions assuming we can understand the other's mental state. Some have argued that the theory of mind ability may give rise to greater levels of manipulation and intentional deception of others (Frith \& Frith, 2005); the idea here is that if I have a sense of what you know, I can decide what to share, in good faith or not. However, others have suggested that those with high theory of mind ability are actually less likely to engage in manipulation of others as they tend to score higher on empathy (Lyons et al., 2010).

There are some studies that have considered the ways that people engage theory of mind with computers (Kramar, von der Putten, \& Eimler, 2012; Rilling, Sanfey, Aronson, Nystrom, \& Cohen, 2004; Thomas, 2001), but it is surprising how little work has been done in this area given people's likelihood to engage with machines socially. The work of Rilling et al. (2004) suggests that interactions with computer partners activate parts of the brain associated with theory of mind, although the effect is not as strong as it is for interactions with human partners. Nonetheless, Functional magnetic resonance imaging (fMRI) data suggests that computer interactions can stimulate some theory of mind behaviors (Rilling et al., 2004). We posit that people use theory of mind as an important tool to negotiate their customer service experiences via IVRs:

$\mathrm{H} 2$ : Users will employ theory of mind when engaging with IVR technology.

\section{Methods}

As this was an exploratory study, we conducted structured interviews and focus groups in order to gain insight into our research question and hypotheses. Our team aimed to see how people managed client service contacts given their limited power to bypass the IVR gatekeepers. Fifty interviews were conducted either in person or phone by the primary investigator over the course of a two-month period in the summer of 2015. The goal of the interviews was to ask about participants' general attitudes towards client service and IVR generally, as well as their specific experiences with certain client service platforms such as speaking with a live agent, using live chat, etc. IVRs, in the form of automated virtual agents and other voice controlled assistants like Siri, were discussed in-depth in order to assess knowledge and comfort with the technology as well as to understand how they use, or strategically opt out of using, the IVR technology. IVRs, robots, and computers were not explained to participants via explicit definitions. Participants did not ask for clarification or definitions of these terms during 
the interviews or focus groups, and they often used IVRs, robots, and automated computer systems synonymously when expressing their experiences. They were provided up front with the consistent idea of IVRs: in the realm of customer service, they would have to interact with a predefined or automated system. This was all with the general framing that they are interacting with non-human devices. Interviews ranged from 15 minutes to an hour, with the average about 25 minutes. All interviews were audio recorded.

Three focus groups were conducted in order to test these topics in a group setting. Focus groups were an integral component of this work because they provide insight into "not only what people think but how they think and why" (Kitzinger, 1995, p. 299). The focus group leader did not need to ask many questions to stimulate intense discussion among participants: everyone had memorable client service experiences that they wanted to share. This afforded the opportunity to listen for the moments of consensus in their experiences. Focus groups allow "co-construction of meaning" (Tiggemann, Gardiner, \& Slater, 2000, p. 646), and opportunities for participants to question each other (Neuman, 2006), both of which provided deep insight into this topic. For example, in each group there was a significant debate about the experience of talking to automated systems during these calls, and particularly whether the IVR robot can or should express sympathy. The discussions in the groups were far richer than those in the interviews because people could question each other's assumptions and understandings. All groups were audio recorded and ranged between 45-75 minutes in length.

\subsection{Demographics and Recruitment}

Participants for both the focus groups and the interviews were recruited via snowball sampling and using the researchers' own personal networks. Despite its obvious weakness, this recruitment strategy nonetheless follows early work on the internet that recruited through local personal networks (Kraut et al., 1998). However for our purposes we advertised the study via several researchers' social media platforms. No incentives were offered for participation, although food was provided for focus group participants. Every effort was made to recruit a representative sampling of people based on age, race/ethnicity, and gender. Granovetter's (1973) concept of the strength of weak ties was employed to obtain access to new opinions because weak ties offer entrée to people in different positions in the social network, who will most likely possess different information and ideas.

Focus group recruitment was targeted so that the groups were clustered by age and degrees of selfassessed skill and comfort with technology. The first group $(n=6)$ comprised young (ages 19-29) heavy tech users and had four women and two men; the second group $(n=7)$ was older (60s and 70s) and involved low to medium technology users, with four men and three women. The final group $(n=8)$ included four men and four women in their 30s and 40s and was the only group that included some mix of technology use; there were some professionals who were deeply connected to their technology and early adopters of new features and devices. Others were more ambivalent about technology and/or relied on only a few key features such as text messaging and email.

\subsection{Data Analysis}

The data collection and analysis was rooted in the grounded theory approach (Glaser \& Strauss, 1967). We allowed the theory to emerge from the data and focused on extensive use of note taking and memo writing during the data collection and analysis processes. The primary investigator took field notes during all interviews and focus groups. After each interview or focus group she wrote memos (Covan, 2007; Glaser \& Strauss, 1967) to keep track of emerging themes and links within the data.

Upon completion of the data gathering phase the primary investigator listened to all of the recordings of the data and supplemented her notes and memos as necessary. These analytic memos and notes were combined to create theoretical memos based on Glaser's (1998) idea that themes that emerge from data analysis can build theory. To accomplish this, memos were created for each emerging theme, with relevant supporting evidence added from the analytic memos. This enabled us to see which theories and concepts were most robust. Theories were identified based on the previous literature. For example, to identify whether theory of mind was present, the primary investigator analyzed the transcriptions for instances of the participants reporting perceived deception, motive, and intent of the IVR technology.

\section{Findings}

Participants reported that they contact client services for a variety of reasons, anything from making dinner reservations to concerns over bank or internet fraud; however, they did note that in the vast majority of situations they are emotionally invested in the outcome. People spoke about times when they had no power/heat during a winter storm, losing internet connection during exam time, and contacting companies about concerns about fraudulent charges or identity theft. In all of these cases the experience, regardless of the client service they receive, is fraught and stressful. Unsurprisingly then, people valued a speedy resolution above all else in their client service experiences. In these cases, clients are especially helpless; they cannot cancel their bank card themselves or fix the electricity or Wi-Fi in their homes; they are at the mercy of the companies.

Additionally, people who spoke about less dramatic issues, calling about a defective product or cancelling subscriptions, still reported feeling stress about their position of relatively limited power in the interaction. As 
one participant in his 30s reported, "frustration mounts as time ticks away" without a resolution. Even for mundane activities such as cancelling a newspaper subscription, which two participants referenced, the frustration stems from the fact that they cannot handle the situation on their own and feel like they are beholden to the client service agent or IVR for resolution.

Because of their feelings of urgency, emotional investment, and perceived helplessness in the client service experience, contact with a live client service agent is the most sought after channel of client service communication, with many referring to it as "instinct" or "habit" to call to speak to a person. While it is the most sought after, the IVR feature that usually accompanies calls to companies received the most complaints from participants. People talked about long waits on hold and challenges in getting directed correctly by the IVR, but reported that they are willing to deal with this because they hold onto a belief that the live representative will help them. As one woman in her mid-20s said, "I feel like I can convince a person to help me". Two reported that they just prefer to complain to a live person. A young man in his 20 s said that if he doesn't "have a person it feels like you are really on your own". Another female in her 20 s said that she prefers to deal with a person instead of an IVR robot because "the robot has no sense of urgency. The robot doesn't care about me", which is an interesting comment to consider in light of the literature on human subconscious social responses to computers (Nass et al., 1996; Nass \& Brave, 2005; Reeves \& Nass, 1996). In this case, it would seem that the IVR robot has not been personified in a humanistic way, as she assumes it is less concerned or empathetic than a human would be. This of course does not imply that she did not respond socially to the IVR during the call, but rather to suggest that she sees a difference in her likelihood of success between the IVR and the live agent interactions. There is inherently more trust that the live representative will "see it through" as one respondent put it, compared to the IVR.

In addition to placing more trust in the human interactions, participants also noted that they are more likely to praise or think highly of a client service experience with a person as opposed to an IVR. The millennials in the tech savvy focus group all agreed that they will only "rave" about client service from a live agent because "I don't think anything of it if it's a robot". In other words, the IVR robot accomplishing client service seemed to be nothing special. They also noted that you can only get perks (e.g., free shipping, discounts) by phone because the agents are "invested in satisfying people right then and there". While this group agreed that the IVR can provide the efficiency that they seek in client service interactions, they noted that a robot technology will not garner "rave" reviews from them that a person would. Based on this it would seem that this group of millennials will discount robot technology to some degree. Again, the robot interaction is expected, or perhaps even unnoticed, in a way that interactions with live agents are not.
People see the live agent call as the final arbiter of their client service fate. As such, the IVRs that initiate these calls to a live agent were described as barriers to this service, a "roadblock" to getting help as one participant described it. Others described the time with IVRs as "feel[ing] like I'm being managed". A woman in her 30 s said that the IVR was just "dragging the conversation out...you're just in line waiting", and she went on to say that the IVR does not actually lead to faster resolutions. It is clear from participants' language that they do not believe that the IVR will resolve their problems, and as such, they are things that must be endured to get through to the live agent.

And enduring them is hard work. People reported long wait times, questions that were irrelevant to their specific needs, and being "stuck in the loop of pressing buttons". In addition, the IVRs can be frustrating because of their long, but largely unhelpful, menu options. A woman in her 50s said that when there are too many menu options given verbally by the IVR, it is hard to remember and organize her options. In the third focus group (30-40 year olds) many noted that none of the menu options are relevant and "you can never remember which one" to press. These respondents said that they feel pressure in these situations because if you press the wrong button you will lose your place and probably have to restart the call. No one had the sense that they had the power to rectify a mistake in their IVR selections, which only further exacerbates levels of stress and frustration.

People also spoke about their frustration with company specific "keywords" or the "language" that IVRs employ. When the IVRs ask people to explain/state their problems, people were happy to have the chance to articulate their problems instead of pressing buttons, however, because of the robotic nature of the exchange many in the interviews talked about the importance of understanding the "language" or "keywords" of the IVR, which they noted can be hard to figure out. All focus group participants agreed with this as well. One woman in her 30s reported that she was frustrated because "I can't just tell my story" to the IVR in an open-ended way. And people expressed a disinclination to learn; as one woman in her 40s reported, "I don't want to have to know the language of the bank. I want the bank to know my language". This language is particularly hard to learn because of the infrequency of client service contact; one woman in her late 30s said that "I don't call these things enough to have their language be my language". Connected to this notion of language, some people noted that the IVRs can be challenging to deal with because they require the client to think about how to phrase the problem or question before speaking. One man in his 40s reported that he finds himself "verbally farting" when he tries to verbalize his problem to the IVR. Others reported the stress they felt when they do not really know the problem (i.e. trying to verbally explain a pipe or wiring problem in the house). These are real chal- 
lenges for IVRs, which have a hard time following contradictions or stops/starts in language with accuracy.

This then introduces another layer to the power dynamic as the client must negotiate an IVR gatekeeper that is confusing to engage with because of long menus and unique keywords and language. Many respondents felt that the confusion, coupled with the long wait times, feels intentional; one referred to it as "strategic waiting" and others noted that it feels like "they don't want you to get through" to a live agent. We found that to manage this people have developed strategies for negotiating their IVR contacts that they alter depending on their experiences with the system in place. Thus, we see a difference between initial engagement with a company's IVR and the strategies they begin to employ as "time ticks away" and/or they become familiar with the IVRs language.

\subsection{Initial Contact: On Guard but Polite}

With the exception of a few people who reported that they never even listen to a IVR robot, opting out as soon as possible, most people reported that they will "entertain" the robot when they first hear it. Their reaction to their initial interaction with the robot seems to be to employ their social skills and work with the IVR to accomplish their goal. People used language that suggested that they are accommodating the machine, saying that they will "try it out", "try to listen", or "tolerate" the IVR when they first begin their client service experience. In these moments they will try to follow social norms and "engage" with the IVR. However, this moment of polite interaction with the IVR is short lived. Several people talked about how they had a tolerance for listening to only a certain number of prompts. One man in his $30 \mathrm{~s}$ noted that "if I'm not at the right place in 2 or 3 beats, I'm not willing to keep trying things on a menu". Harkening back to the need for a quick resolution to their client service issue, people are only willing to interact politely with the IVR software for a short amount of time. However, again they cannot be rid of the IVRs altogether as they still need the robot to connect them to the live agent.

\subsection{Continued Contact: Strategic Outmaneuvering}

Once people have exhausted their patience with the IVRs they employ new strategies to manage the roadblock. Some reported that they will immediately start saying "agent", "representative", or pressing 0 in an effort to try to bypass the IVR robot. One woman in her 30s reported that she pretends "not to speak English or I say I have a rotary phone" so that she gets directed to the live agent. Interestingly in this phase they are not "tolerating" or "engaging" the IVR, but have instead moved to yelling at the robot, interrupting it, and/or pressing random buttons.

These experiences of increasing frustration and the strategic work-arounds they employ are remembered by these participants and they in turn inform their future client service interactions. With the exception of a few of the older participants who said that they always call client service out of "habit", most other people were willing to use different platforms to get to their end goal of a personalized, fast response. In fact, most participants were strategic in the channels they selected based upon 1) their prior experiences with the company's client service (i.e. knowing that the phone is notoriously slow) and 2 ) the nature of their question (ambiguous or multistep vs. straightforward; urgent vs. less urgent). In cases that they perceive to be ambiguous, involve a multistep process, and/or are urgent they prefer to call, while they may opt for email or live chat with straightforward questions. All participants reported that they consider these questions before deciding upon the channel to utilize. However it is important to note that a minority of participants noted that the technology channel they used is often preselected for them by the company (i.e. the company only offers an email or a web form). Thus the company can dictate the channels.

In most cases though, people are open to exploring other platforms as a way to optimize their results. First, every participant reported that he/she initiates any customer service contact by visiting the company website. Most navigated through the website a bit first, either to look for the customer service phone number (particularly popular with older respondents), or to search through the FAQs for an answer to their problem in an effort to avoid customer service altogether.

Others reported collective strategies that were beneficial both in terms of resolving their customer service issue as well as providing a psychological benefit. Some clients (40s and under) reported Googling their problem or question or looking on Twitter or Reddit to see how others are talking about this issue online. They liked doing this because it may help to lead them to a resolution. However, even if the search did not yield an answer, they reported that the results are still helpful because it puts the situation in perspective for them. The focus group of 30-40 year olds agreed with this, and as one male interviewee reported, it is helpful to know that others have had the problem too because it gets him out of thinking "why do these things always happen to me?" The focus group of tech savvy individuals picked up on this theme, with many noting that they look on Reddit to see that others are having the same problem and that it "keeps my emotions in check" to know that "others out there" have the same problem.

In general, what we see is participants' willingness to engage with the IVRs shifts as people gain more knowledge and understanding of how these processes will play out. While they may be willing to engage the IVR at the beginning, the perception of IVRs as gatekeepers means they have to create a work around to the system that is set up. They do this in a variety of ways, but most often either try to bypass the robot in the system or try another platform. Again, these strategies are devised solely to get a fast resolution to their often stressful client ser- 
vice problems. As such, they make a calculated decision based on the importance of their problem and the ease of explanation. The more serious and confusing problems lead most people to call for a live agent, because the perception is that the live agent is most capable of solving the problem. Or at the least, the call will not end without some sort of response and understanding. For many people, just knowing that someone was working on a problem was enough to make them feel okay, but the perception is that only a person can create this feeling, not a robot.

\subsection{Programmed Politeness}

Many people in the interviews and focus groups made reference to the fact that occasionally they will encounter an IVR that engages in a programmed politeness, i.e. in its stilted robot voice the IVR apologizes to the client or expresses sympathy for the problem. This experience proved to have mixed effects with participants. The tech savvy focus group spent a great deal of time discussing this issue and largely agreed that it was possible that a robot could sympathize, or that someone could "program in sympathetic words" to make the overall experience more positive. In contrast several interviewees and participants in the focus group with 30-40 year olds said that they dislike the "fake empathy" of the robot. In both of these focus groups there was real debate about whether it mattered if the IVR was sympathetic as long as the problem was resolved, but there was no definitive answer on this as some felt that the resolution is the only thing that matters while others said that good customer service could improve any call.

We would like to acknowledge the limitations within the study. This was a small, exploratory study consisting of three focus groups and fifty interviews with time constraints. We have no claims to universality, as we used a non-random convenience sample and snowball method for recruitment. However, the data we gathered points very clearly to generalizability and it seems extensible. We hope to examine this further in future studies.

\section{Discussion and Conclusion}

This study explored how users manage their position communicating with IVR client service interfaces, with a focus on users' perceived amount of power. Our work first builds off of Nass et al.'s theories (1996, 2000, 2005) and those of Halpern and Katz (2013) and Katz and Halpern (2013) concerning social interactions with computers. Nass et al.'s work (1996, 2000, 2005) revolves around the media equation theory, the idea that people treat and behave toward computers the same way that they would another human. Research by Halpern and Katz (2013) and Katz and Halpern (2013) examines how users perceive robots and human-robot interaction. Similar to Nass et al., Katz and Halpern (2013) found that humans will ascribe humanlike qualities to robots even if they do not have a human-like appearance. However, the researchers also found that a user's willingness and likelihood to accept interaction with a robot is not dependent on the robot's human-likeness (Halpern \& Katz, 2013).

Our findings echo aspects of these ideas of projecting human-like qualities onto IVR robots, specifically within initial interactions. $\mathrm{H} 1$ predicted that users interacting with IVRs for client service help will follow social norms and engage in polite interactions. We found this hypothesis to be partially supported, dependent on the users' experience with the IVR technology. What we saw from our studies is that, in novel IVR interactions, people revert to their social skills and human routines. At first, users are polite and willing to "engage" with the robot. Respondents indicated they would "try to listen" or "test out" the IVR service offering, which suggests that they are accommodating the system as if it were a live agent. In these cases, the users have limited power because the IVR is the gatekeeper and the situation is novel. People expressed frustration when engaging with these IVR technologies for the first time because the systems often required the understanding of "keywords" or the "language" of the automated system. Numerous study participants indicated that the infrequency of their customer service contact made it hard to learn the IVR's language and keywords. In these scenarios, there is a power imbalance between new IVR users and the IVR technology. Ultimately, to Castells' (2009) point, the protocols dictate the communication. As such, users rely on politeness and cooperation as a negotiating skill; they will listen through the interactions because they lack the power, in this sense the knowledge of an IVR system, to speed through the IVR prompts. This also provides insight into RQ1, which asked whether a users' behavior toward IVRs would change based on their perceived amount of power.

Through more frequent interactions, however, and as the conditions change and the IVR experience is no longer novel, people begin to see the technology only as a tool (i.e. less personal), specifically a tool that can be managed. Study participants indicated that, once they familiarized themselves with the IVR-friendly language and knew which buttons to press sequentially, IVR became less "stressful" and, in turn, "easier". Users stated they felt in "control" as they were able to easily go through the prompts and skip over irrelevant options after becoming familiar with the technology. With this reframing of the IVR as a merely a tool, people devise ways to work around the gatekeeper and begin to take control of the situation. They do this by dodging the IVR protocols by learning shortcuts they can apply to all IVR technology. These include pressing 0 , yelling for an agent, or by turning the experience into a collective action whereby they search for human help online to bypass customer service altogether. By engaging in these behaviors, the clients develop more negotiating power as they no longer entirely unequal with the IVR technology.

With regards to the idea of IVR devices exhibiting emotion, empathy from computers was reported as 
seeming "fake" and laden with ulterior motives. This perception of the technology's insincere empathy becomes particularly detestable in this second phase as it feels like a power play from the company. The idea is that this "programmed politeness" is a tool used by the companies to limit anger and discourage future action from users. The politeness is programmed to affect a certain outcome in the negotiation and it feels abhorrent to clients because it is false empathy, not honest or benevolent in intention. These ideas support $\mathrm{H} 2$, and users do employ theory of mind when interacting with IVR technology. This notion of the IVR being manipulative and disingenuous ties in with aforementioned research on cognitive science, specifically the theory of mind, which examines how people assign varying qualities and mental states to others (Kramar et al., 2012; Premack \& Woodruff, 1978; Rilling et al., 2004; Thomas, 2001). The client's theory of mind encompassing IVR technology states that the robot is disingenuous and manipulative. Clients perceive the IVR's empathy as a way to deter future interactions and limit potential frustration: a company's deceptive manipulation tactic. From the user's perspective, once an IVR technology becomes a tool, it really cannot show true empathy.

Overall the customer service IVR experience is an interesting example of the ways in which people approach networked communication structures when they are powerless. What we found is that people are resourceful and able to develop means to enhance their position in the IVR negotiations. The interesting catalyst to these strategies is the reframing of the IVRs as a novel technology to an inhuman tool that must be circumvented. Our study also began to shed light on how media equation theory and theory of mind can be applied to human-robot interaction and perceptions of IVR technology. These vectors of social interaction analysis can be fruitfully expanded with an eye towards not only more fully understanding the social setting of an increasingly technologically mediated world but also to inform more humane usable interfaces to serve people more effectively.

\section{Acknowledgments}

The authors would like to thank Interactions LLC for sponsoring this research.

\section{Conflict of Interests}

The authors declare no conflict of interests.

\section{References}

Castells, M. (2009). Communication power. Oxford: Oxford University Press.

Center for Research on the Information Society. (2015). Modalities of customer service (CRIS Report). Morristown, NJ: CRIS.
Covan, E. K. (2007. The discovery of grounded theory in practice: The legacy of multiple mentors. In A. Bryant \& K. Charmaz (Eds.), SAGE handbook of grounded theory (pp. 58-79). Thousand Oaks, CA: Sage Publications. doi:10.4135/9781848607941.n2

Fairhurst, G. T., \& Chandler, T. A. (1989). Social structure in leader-member interaction. Communication Monographs, 56(3), 215-239.

Foucault, M. (1984). The history of sexuality (Vol. 1). New York, NY: Pantheon Books.

Frith, C., \& Frith, U. (2005). Quick guide: Theory of mind. Current Biology, 15(17). doi:10.1016/j.cub.2005. 08.041

Glaser, B. G. (1998). Doing grounded theory: Issues and discussions. Mill Valley, CA: Sociology Press.

Glaser, B. G., \& Strauss, A. L. (1967). The discovery of grounded theory: Strategies for qualitative research. Chicago, IL: Aldine Publishing Company.

Goffman, E. (1959). The presentation of self in everyday life. New York, NY: Anchor Books, Doubleday.

Granovetter, M. S. (1973). The strength of weak ties. American Journal of Sociology, 78(6), 1360-1380.

Groom, V., Srinivasan, V., Bethel, C., Murphy, R., Dole, L., \& Nass, C. (2011). Responses to robot social roles and social role framing. International Conference on Collaboration, Technologies, and Systems. doi:10.1109/CTS.2011.5928687

Halpern, D., \& Katz, J. E. (2013). Close but not stuck: Understanding social distance in human-robot interaction through a computer mediation approach. Intervalle, 1(3), 17-34.

Katz, J. E., Aspden, P., \& Reich, W. (1997). Public attitudes toward voice-based electronic messaging technologies. Behaviour \& Information Technology, 16(2), 17-39. doi:10.1080/014492997119860

Katz, J. E., \& Halpern, D. (2013). Attitudes toward robots suitability for various jobs as affected robot appearance. Behaviour \& Information Technology. doi:10.1080/0144929X.2013.783115

Kitzinger, J. (1995). Introducing focus groups. British Medical Journal, 311(1), 299-302. doi:10.1136/ bmj.311.7000.299

Kramar, N., von der Putten, A., \& Eimler, S. (2012). Human-agent and human-robot interaction theory: Similarities to and differences from human-human interaction. In M. Zacarias \& J. Valente de Oliveira (Eds.), Human-computer interaction: The agency perspective (pp. 215-240). Berlin: Springer-Verlag.

Kraut, R., Patterson, M., Lundmark, V., Kiesler, S., Mukopadhyay, T., Scherlis, W. (1998). Internet paradox: A social technology that reduces social involvement and psychological well-being? American Psychologist, 53(9), 1017-1031. doi:10.1037/0003066X.53.9.1017

Lyons, M., Caldwell, T., \& Shultz, S. (2010). Mind-reading and manipulation-is Machiavellianism related to theory of mind? Journal of Evolutionary Psychology, 8(3), 261-274. doi:10.1556/JEP.8.2010.3.7 
Nass, C., \& Brave, S. (2005). Wired for speech: How voice activates and advances the human computer relationship. Cambridge, MA: MIT Press.

Nass, C., Fogg, B. J., \& Moon, Y. (1996). Can computers be teammates? International Journal of HumanComputer Studies, 45(6), 669-678. doi:10.1006/ ijhc.1996.0073

Nass, C., \& Moon, Y. (2000). Machines and mindlessness: Social responses to computers. Journal of Social Issues, 56(1), 81-103. doi:10.1111/0022-4537.00153

Neuman, W. L. (2006). Social research methods: Qualitative and quantitative approaches (6th ed.). Boston, MA: Pearson, Allyn and Bacon.

Premack, D., \& Woodruff, G. (1978). Does the chimpanzee have a theory of mind? Behavioral and Brain Sciences, 4(1), 515-629. doi:10.1017/ S0140525X00076512

Reeves, B., \& Nass, C. (1996). The media equation: How people treat computers, television, and new media like real people and places. Stanford, CA: Center for the Study of Language Information and Cambridge University Press.
Rilling, J. K., Sanfey, A. G., Aronson, J. A., Nystrom, L. E., \& Cohen, J. D. (2004). The neural correlates of theory of mind within interpersonal interactions. Neuroimage, 22(4), 1694-1703. doi:10.1016/ j.neuroimage.2004.04.015

Thomas, J. C. (2001). Collaborative innovation tools. In T. Terano, T. Nishida, A. Namatame, S. Tsumoto, Y. Ohsawa, T. Washio (Eds.), New frontiers in artificial intelligence: Joint JSAI 2001 workshop post-proceedings (pp. 27-34). Shimane: Springer.

Tiggemann, M., Gardiner, M., \& Slater, A. (2000). “I would rather be a size 10 than get straight $A^{\prime} s^{\prime \prime}$ : A focus group study of adolescent girls' wish to be thinner. Journal of Adolescence, 23(6), 645-659.

Tjosvold, D. (1984). Cooperation theory and organizations. Impact Factor, 37, 743-767. doi:10.1006/ jado.2000.0350

van Dijk, J. A. G. M. (2010). Review of "Manuel Castells, Communication Power". Communications, 35, 485-489.

\section{About the Authors}
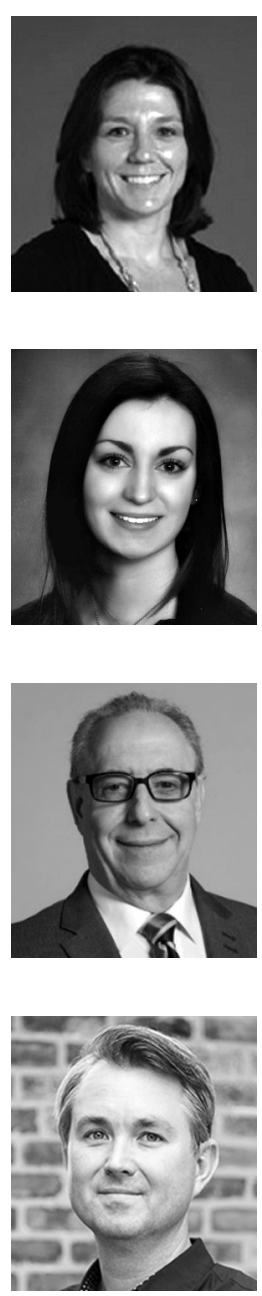

Jill Walsh's research revolves around media usage, particularly social media. She obtained a PhD in Sociology from Boston University in 2014, earlier earned a Master's in Public Policy from Brown University, and a BA from Harvard University. Prior to completing her graduate coursework, she taught, coached, and mentored high school students at the Noble and Greenough School for five years.

Brittany Andersen is a PhD student in the Emerging Media Studies Program (EMS) at Boston University College of Communication. At BU, her research focuses on user experience, mainly how users perceive, evaluate, and respond to different types of media and technology. She received her Master's from the EMS program in 2015, and has experience working on industry research and analyst relations teams.

James E. Katz, PhD., Dr.H.C., is the Feld Family Professor of Emerging Media at Boston University's College of Communication where he directs its Center for Mobile Communication Studies and Division of Emerging Media. He also serves as Distinguished Professor at the School of New Media School at Peking University. His research on social media and mobile communication has been internationally recognized and translated into many other languages. Among his books are Machines That Become Us and the Handbook of Mobile Communication.

Jacob Groshek is an Associate Professor of Emerging Media Studies at Boston University. His areas of expertise concern online and mobile media technologies as their use may relate to socio-political and behavioral health change at the macro and micro levels, and also include analyses of media content and user influence in social media. He uses a blend of interpretive as well as relatively advanced statistical tools for network analysis, forecasting, and explaining where and how the use of media has shaped the course of social, political, and health activity. 